\title{
NutriNet-Salud México. Estudio prospectivo por Internet: 2018-2028. Relación de nutrición y salud y factores determinantes de los hábitos alimentarios y el estado nutricional
}

\author{
NutriNet-Salud México. Prospective study online: 2018-2028. Relationship between \\ nutrition and health and determinants of dietary habits and nutritional status
}

Jorge Armando Barriguete-Meléndez ${ }^{1,2,3 *}$, Serge Hercberg², Pilar Galán ${ }^{4}$, André Parodi5 ${ }^{5}$ Jacques Baulieux ${ }^{5}$ y RED Nutrinet-Salud México ${ }^{6}$

${ }^{1}$ Instituto Nacional de Ciencias Médicas y Nutrición; ${ }^{2}$ Consejo Estratégico Franco Mexicano; ${ }^{3}$ Academia Mexicana de Cirugía. Ciudad de México, México; ${ }^{4}$ Université Sorbonne Paris Cité; ${ }^{5}$ Académie Nationale de Médecine. París, Francia; ${ }^{6}$ RED Nutrinet-Salud México (ver agradecimientos).

\begin{abstract}
Resumen
Introducción: NutriNet-Salud México es un sistema de información en salud digital, instrumento de e-epidemiología, en línea, abierto y gratuito, para el registro y el análisis de los factores determinantes de los hábitos alimentarios y el estado nutricional de la población mexicana, para la prevención del sobrepeso, la obesidad y las enfermedades crónicas no transmisibles para el período 2018-2028. Objetivo: Describir el diseño, el desarrollo y la implementación de NutriNet-Salud México a partir del modelo francés NutriNet-Santé France 2008-2018. Método: La plataforma NutriNet-Salud México es la base digital para el desarrollo de un sistema de información en salud -para un estudio de cohorte prospectivo programado para 10 años (2018-2028) - con un sitio web dedicado cuyo desarrollo permite tener varias poblaciones de estudio que responden un conjunto inicial de cinco cuestionarios autoaplicables validados en población mexicana. Resultados: La información obtenida permitirá desarrollar investigación aplicada, conocer y vigilar los aportes alimentarios y el estado nutricional de la población, evaluar el impacto de acciones de salud pública sobre el comportamiento alimentario y el estado nutricional, y comparar poblaciones entre países (México, Francia, Bélgica y Suiza) e institutos nacionales, universidades y Estados. Conclusiones: NutriNet-Salud México permitirá coadyuvar en investigación y acción pública, especialmente en la orientación de políticas públicas de México en materia de nutrición. Los elementos científicos aportarán recomendaciones nutricionales adecuadas a diferentes poblaciones, y permitirán acceder a una representativa muestra poblacional nominal a bajo costo y en tiempo real con doble abordaje de e-epidemiología: estudio de cohorte para identificar causalidad y estudios transversales periódicos (investigación descriptiva, monitoreo y evaluación).
\end{abstract}

PALABRAS CLAVE: NutriNet-Salud México. Sistema de información en salud. Nutrición. Obesidad. Enfermedades crónicas no transmisibles.

\begin{abstract}
Background: NutriNet-Salud Mexico is a digital health information system, e-epidemiology instrument, online, open and free, to recording and analysis the determinants of dietary habits and nutritional status of the Mexican population, for the prevention of overweight, obesity and noncommunicable diseases for the period 2018-2028. Objective: Describe the design, development and implementation of NutriNet-Salud Mexico from the French model NutriNet-Santé France 2008-2018. Method: NutriNet-Salud
\end{abstract}

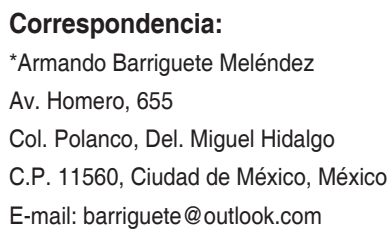

Fecha de recepción: 24-01-2017

Fecha de aceptación: 13-09-2017

DOI://dx.doi.org/10.24875/CIRU.M18000011
Cir Cir. 2018;86:8-19

Disponible en PubMed www.cirugiaycirujanos.com 
Mexico platform is the basis for the development of health information system for prospective cohort study, scheduled for a period of 10 years (2018-2028), with a dedicated website, and its development will enable to have multiple study populations within an initial set of five self-applicable questionnaires validated in Mexican population. Results: The information will enable to develop applied research, learn and monitor food contributions and nutritional status of the population, assess the impact of public health actions on feeding behavior and nutritional status, comparing populations between countries (Mexico, France, Belgium and Switzerland) and national institutes, universities and states. Conclusions: NutriNet-Salud Mexico will provide information for assist in research and public action, especially to guide public policies on nutrition Mexico. The scientific elements will make appropriate nutritional recommendations to different populations and access to a representative nominal population sample with low-cost, in real-time, and with dual approach to e-epidemiology: cohort study to identify causality and cross-sectional studies (descriptive research, monitoring and evaluation).

KEY WORDS: NutriNet-Salud México. Health information system. Nutrition. Obesity. Noncommunicable diseases.

\section{Introducción}

Las enfermedades crónicas no transmisibles relacionadas con la nutrición y la obesidad, como la diabetes mellitus tipo 2 (DM2), la hipertensión, las enfermedades cardiovasculares (ECV) y el cáncer, representan en muchos países un importante problema de salud pública debido a los altos índices de morbilidad y mortalidad, y los gastos para su atención médica. Cada año, 38 millones de personas mueren en el mundo a causa de a estas enfermedades. Más de 14 millones de las muertes por enfermedades crónicas ocurren entre los 30 y los 70 años de edad, y de ellas, el $85 \%$ sucede en países en desarrollo. En México, las ECV, la DM2 y los cánceres representan más del $50 \%$ de las muertes anuales ${ }^{1}$.

La obesidad es un problema grave de salud en México $^{2}$. En la actualidad, aproximadamente el $70 \%$ de los adultos de más de 20 años, es decir, alrededor de 49 millones de personas, padecen sobrepeso u obesidad $^{3}$, así como el $35 \%$ de los menores en edad escolar (cerca de 12 millones). La Encuesta Nacional de Salud y Nutrición (ENSANUT) de $2012^{2}$ identifica una prevalencia del $9.2 \%$ de la población adulta en México (alrededor de 6.6 millones) diagnosticada de diabetes mellitus; sin embargo, la prevalencia alcanza cerca del $14 \%$ si se incluyen los individuos $\sin$ diagnóstico ${ }^{3}$.

Tan solo en 2008, el costo total de la obesidad fue calculado en 67,000 millones de pesos, costo directo anual en atención médica para el sistema público de salud de las 14 complicaciones derivadas de cuatro de las enfermedades vinculadas con la obesidad: DM2, ECV, cáncer de mama y osteoartritis. De seguir la misma tendencia, los costos podrían alcanzar en 2017 entre 151,000 millones y 202,000 millones de pesos $^{4}$.
Las enfermedades crónicas son de etiología compleja, y los factores genéticos, biológicos, conductuales y ambientales desempeñan un papel importante. Factores de vida modificables, como la actividad física, la duración del sueño, los hábitos alimenticios y el consumo de alcohol y tabaco, contribuyen considerablemente al desarrollo de las enfermedades crónicas más comunes y mortales.

Dado que estos problemas tienen su origen generalmente en los primeros años de la vida, y se mantienen y refuerzan con la edad, es importante desarrollar intervenciones de manera oportuna, constante y a largo plazo, que no solo promuevan hábitos saludables, sino que permitan a las personas aprender a observarse, descubrirse y conocerse a través de sus propias conductas. De ahí el interés de poder seguir de manera nominal a grandes poblaciones durante largos períodos de tiempo (al menos de 5 a 10 años), con perfiles diferentes y en plataformas digitales seguras que permitan análisis complejos y respeten la confidencialidad 5 .

Actualmente existen estrategias de prevención que han demostrado su efectividad en el mejoramiento de la salud mediante cambios en los estilos de vida; de manera complementaria, esto ha coadyuvado a la concientización de los hábitos de la población. Sin embargo, es fundamental que los individuos aprendan a tomar decisiones informadas sobre su comportamiento y tratamiento basado en los resultados diarios, y es aquí donde un sistema automatizado de registro puede ser altamente eficiente.

La evidencia científica señala la importancia de desarrollar estudios de cohortes longitudinales, con períodos de observación en el mediano y el largo plazos, complementados con estudios comparativos, para conocer dinámicamente el comportamiento y las diferentes conductas de grandes poblaciones, y así 
identificar los factores de riesgo y los elementos para la protección en salud ${ }^{6-9}$.

En el caso particular de México, la Secretaría de Salud Federal desarrolla e implementa la Estrategia Nacional para el Control y Prevención del Sobrepeso, Obesidad y Diabetes ${ }^{10}$, que integra diversas acciones en tres principales pilares: 1) modificación de los hábitos de la población a través de estrategias de mercadotecnia social en salud y promoción de estilos de vida saludables; 2) refuerzo de la calidad de la atención médica, el manejo y la prevención de la obesidad y la diabetes en el primer nivel de atención, con mayor énfasis en capacitación y actualización del personal médico; y 3) modificación del entorno obesogénico mediante la regulación sanitaria y la política fiscal, reduciendo así la disponibilidad y el consumo de alimentos con alta densidad calórica. Dentro del marco de esta estrategia se insertó el Observatorio Mexicano de Enfermedades No Transmisibles (OMENT), con el objetivo de mejorar la vigilancia epidemiológica de dichas enfermedades, en el que se incluye el monitoreo de la obesidad, para brindar información al público usuario y los tomadores de decisiones con respecto a la evaluación y el desarrollo de políticas públicas.

Por lo anterior, el registro sistemático de las actividades cotidianas y la retroalimentación sustentada en la mejor evidencia posible pueden ser una estrategia adecuada y efectiva para el reforzamiento positivo, el control y la prevención de las enfermedades crónicas no transmisibles, además de servir como guía longitudinal a corto plazo personal, familiar e institucional, y a mediano plazo estatal y nacional. Al generar datos a partir de la observación de hábitos nutricionales de diferentes poblaciones mexicanas, NutriNet-Salud México se convertirá en un aliado estratégico del Plan Nacional y del OMENT.

\section{Método}

NutriNet-Salud México es una plataforma digital en Internet basada en la web 2.0 (Fig. 1) para el estudio de una cohorte prospectiva a 10 años. Implica el desarrollo de un instrumento de e-epidemiología para la creación de un sistema de información en salud en México, basado en el modelo francés de NutriNet-Santé ${ }^{11}$, a través de cuestionarios autoaplicables validados para la población mexicana.

NutriNet-Santé utiliza cuestionarios autoaplicables en línea para identificar y conocer los diferentes grupos poblacionales a través de sus conductas nutricionales y de salud ${ }^{12,13}$, y cruza la información recabada con el acopio de datos biológicos, permitiendo explorar de cerca los hábitos de la población francesa y sus consecuencias ${ }^{14-16}$. De este modo, la plataforma NutriNet-Santé resulta ser un modelo metodológico a consultar y tomar en cuenta para dar seguimiento a

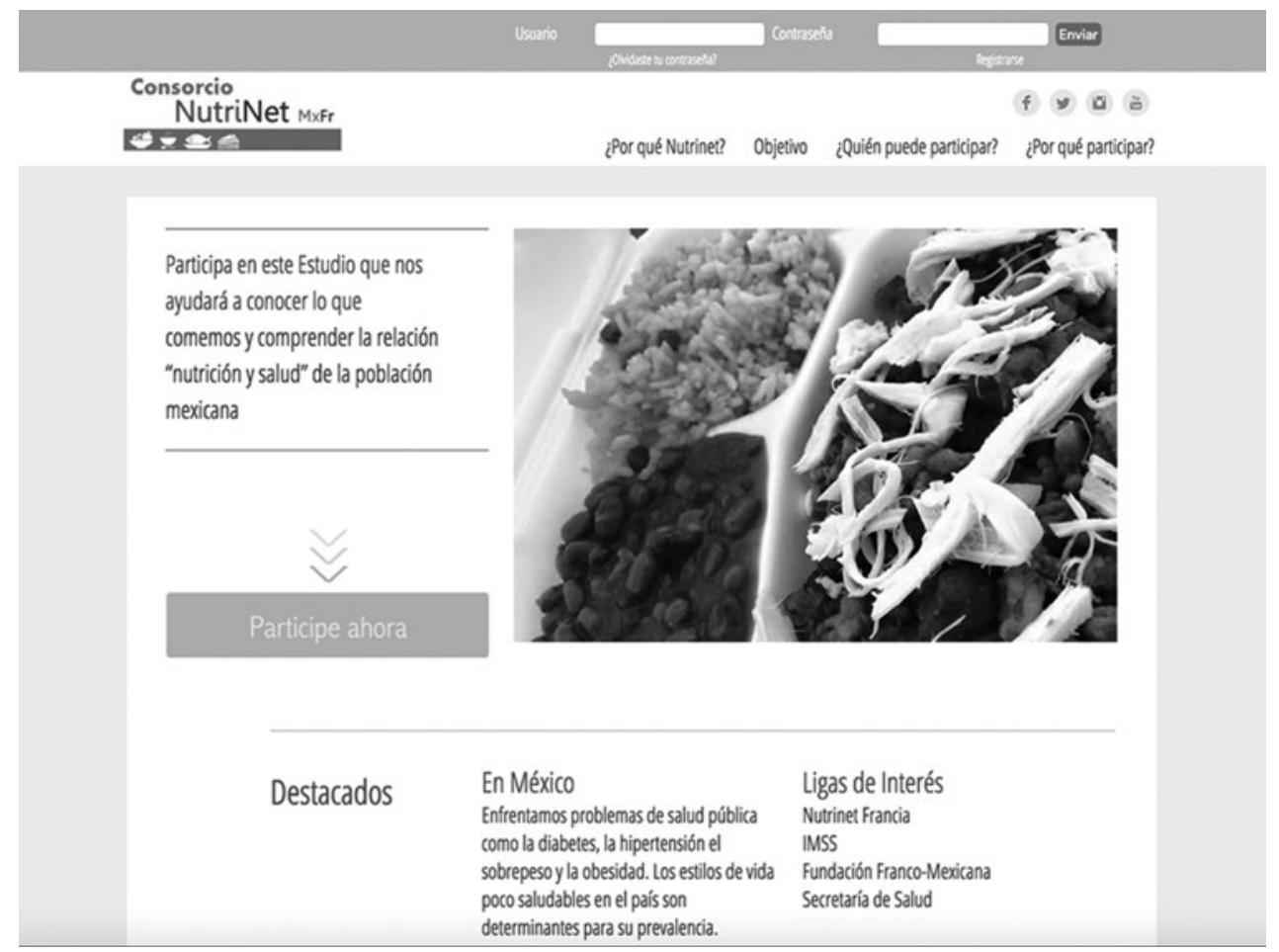

Figura 1. Página de NutriNet-Salud México. 
los hábitos y comportamientos de la población relacionados con la nutrición y los estilos de vida, de gran utilidad en la formulación de acciones y políticas en países emergentes, no solo aplicable para México, sino también para países latinoamericanos a partir de nuestra experiencia.

NutriNet-Salud México ofrece una gran oportunidad de acceder a una muestra representativa poblacional nominal a un bajo costo y en tiempo real, con un doble abordaje de e-epidemiología: un estudio de cohorte que permite identificar la causalidad (investigación etiológica), y estudios transversales que se repiten (investigación descriptiva, monitoreo y evaluación). Brinda además una gran oportunidad para incluir otros estudios de cohortes, beneficiándose del bajo costo, la continuidad y la asociación a las líneas.

En el proyecto NutriNet-Salud México colaboran instituciones académicas, de investigación y de gobernanza en salud. Cuenta con la participación de siete Institutos Nacionales de Salud: Nutrición, Salud Pública, Perinatología, Cardiología, Psiquiatría, Geriatría y Cancerología; tres Universidades: Universidad Autónoma de Guadalajara, Universidad de Guadalajara y Universidad Iberoamericana; dos sistemas de salud estatales: Estado de México y Michoacán; dos organizaciones de la sociedad civil: la Fundación Franco Mexicana para la Medicina IAP y Fomento de Nutrición AC; la Secretaría de Salud Federal y el Instituto Mexicano del Seguro Social (IMSS). Todos participan con colaboraciones definidas en la clarificación de lo que comemos los mexicanos y su construcción nutricional, la identificación de problemas de salud relacionados con la nutrición y el posterior análisis de la información disponible como líneas de investigación de interés de cada institución.

\section{Etapas de desarrollo de NutriNet-Salud México}

La construcción de la plataforma NutriNet-Salud México está planeada en cinco etapas, siguiendo la línea del estudio francés:

\section{Definición de los ejes de investigación prioritarios en salud}

Con el objetivo de estimular el desarrollo de los recursos humanos de alto nivel dedicados a la investigación y promover la vinculación entre la investigación y el desarrollo de competencias técnicas, se trabajó en la definición de los ejes de investigación prioritarios en salud de interés para cada institución, a fin de fortalecer la investigación científica de excelencia, y contribuir a mejorar la salud y el bienestar de la población mediante la generación y la eficiente utilización de nuevo conocimiento.

NutriNet-Salud México está estructurado de tal forma que facilita la difusión y el uso de la información. La estrategia de divulgación y diseminación de información estadística contempla un corte semestral de los registros, con los que trabajan las instituciones participantes como responsables de la elaboración y la difusión de las cifras oficiales, los informes estadísticos y las publicaciones científicas, siempre con aporte a la política pública.

\section{Desarrollo de cuestionarios}

El modelo francés NutriNet-Santé incluye cinco cuestionarios: 1) aspectos económicos, demográficos, sociales y de estilo de vida; 2) salud; 3) antropométrico; 4) actividad física y sedentarismo; y 5) alimentación y formulario de inscripción. Se llevó a cabo el proceso de traducción-retraducción (francés-español-francés) con traductor especializado, revisado por los especialistas, excepto el cuestionario alimentario (véase más adelante) (Fig. 2).

- Cuestionario sobre aspectos económicos, demográficos, sociales y de estilo de vida: se recaba información referente a estado civil, número de hijos y nietos, número y características de los miembros de la familia, actual trabajo (o última ocupación), diplomas, categoría profesional del cónyuge y nivel educativo, antecedentes tabáquicos (tipo, cantidad, duración, tabaquismo pasivo), consumo de alcohol (tipo, cantidad, frecuencia) y salario.

- Cuestionario de salud: la información se recoge en relación con el historial médico, antecedentes, uso actual de medicamentos, suplementos dietéticos, historia médica familiar, causas de muerte de los familiares de primer grado (cuando corresponda) y, para las mujeres, historia obstétrica, embarazo, menopausia, contracepción y terapia de reemplazo hormonal en la menopausia cuando corresponda.

- Cuestionario antropométrico: incluye preguntas relacionadas con la talla, el peso, la circunferencia de la cadera y la circunferencia de la cintura (incluye indicaciones para hacerlo correctamente), además de la historia del peso, la práctica de 


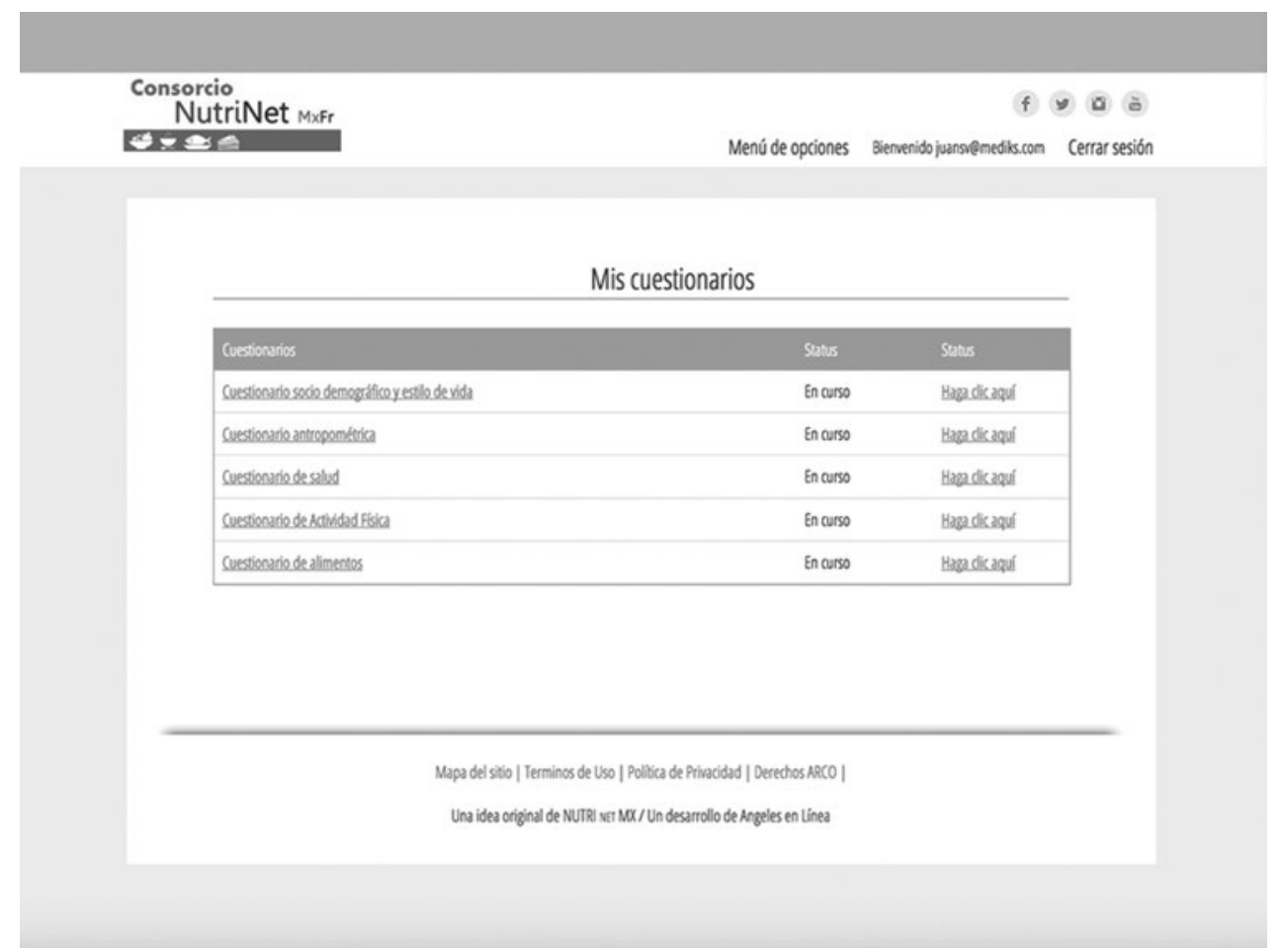

Figura 2. Página en la que se observan los cuestionarios iniciales de NutriNet-Salud México.

dietas restrictivas (historia, tipo y razón) y la autopercepción corporal.

- Cuestionario de actividad física y sedentarismo: se utiliza el cuestionario autoadministrado versión Web del Cuestionario Internacional de Actividad Física (IPAQ) ${ }^{17}$. La actividad física se describe de acuerdo con tres niveles de intensidad de ejercicio (caminar, moderada o vigorosa), la frecuencia de ejercicio a la semana (días por semana) y la duración diaria de cada actividad realizada.

- Cuestionario alimentario: para disminuir el error dado por la variabilidad intrasujetos en el consumo de alimentos, se pedirá que el "nutrinauta» registre 3 días en un periodo no mayor a 1 semana. La información de los 3 días será considerada para el análisis (véase más adelante)

- Cuestionarios adicionales: como parte del programa de seguimiento, los «nutrinautas» completarán cuestionarios adicionales cada año. Al hacerlo, recibirán un correo electrónico automático para informar de la necesidad de completar un nuevo cuestionario en su espacio personal del sitio de Internet de NutriNet-Salud México, asociado a las líneas de investigación propuestas por las instituciones participantes.

- Protocolos auxiliares: en el contexto de protocolos auxiliares que se ocupan de diferentes temas de interés científico, será posible incluir cuestionarios específicos para la población en general o para submuestras elegidas según fenotipos particulares (edad, sexo, región, estado de salud, etc.). Los participantes serán invitados a rellenar este cuestionario una vez que se conectan al sitio en Internet del estudio. La interfaz permitirá a los administradores crear grupos de investigación y determinar si los participantes tienen un perfil o una manera de responder a las preguntas que se inscribe dentro de ciertas categorías, o si corresponden a ciertos criterios de selección para agrupar a los participantes (p. ej., todos los varones mayores de 45 años que no fuman).

Para los instrumentos, a excepción de los cuestionarios de actividad física y sedentarismo, y alimentario, se realizó una prueba piloto en tres grupos de personas, 42 en total, estudiantes, académicos y personal de intendencia, tomando en cuenta el nivel educativo de cada uno. Esta prueba permitió realizar los ajustes pertinentes en cada instrumento y se aplicaron nuevamente los cuestionarios con el mismo grupo, a fin de corroborar si las respuestas concordaban con las de la primera aplicación, y entre los resultados se identificó únicamente un $2 \%$ de promedio de variaciones en cada una de las respuestas.

El cuestionario alimentario de la plataforma NutriNet-Salud México, al igual que NutriNet-Santé en 
Francia, se aplicará con el recordatorio de 24 horas $(\mathrm{R} 24)^{18}$. Este instrumento de evaluación dietética permite evaluar el consumo de alimentos y bebidas durante las 24 horas anteriores a la aplicación del cuestionario. Se planea aplicar tres R24 por cada fase de recolección de datos. La primera ventaja del R24 es que pueden incluirse todos los alimentos y bebidas que cualquier individuo consuma, dado que es un instrumento abierto; la segunda, que permite estimar las cantidades consumidas. Su desventaja es que, al basarse en la memoria del sujeto para reportar lo consumido, hace difícil establecer las cantidades consumidas y muchas veces se sobreestima o subestima el consumo.

Debido a estas limitaciones, se utilizan metodologías para disminuir el error. Una nueva versión que se ha utilizado es el R24 de pasos múltiples, el cual incluye cinco pasos que ayudan al individuo a «repasar» el consumo reportado varias veces para ayudarlo a recordar ${ }^{18}$. El individuo parte de una lista rápida de alimentos y bebidas consumidas, y después va detallando cada una de las comidas que realizó, describiendo todos los alimentos o platillos que registró en cada comida (Fig. 3). Los alimentos se identifican mediante un motor de búsqueda o pueden encontrarse en categorías generales (p. ej., lácteos, bebidas azucaradas, guarniciones, botanas, etc.). Además, para los platillos, el usuario puede añadir ingredientes extra, como salsas, cremas o quesos, que son muy comunes en nuestro país. Para mejorar la estimación de porciones, NutriNet-Salud México incluye herramientas visuales, como fotografías de diferentes porciones de alimentos.

La metodología de trabajo del cuestionario de alimentación de NutriNet-Salud México, se divide en tres etapas:
- Primera etapa: lista de alimentos, platillos y bebidas. Se realizó una selección de alimentos y bebidas de la que partiría el cuestionario, que incluía información nutrimental para poder realizar el análisis del consumo. El Instituto Nacional de Salud Pública (INSP) cuenta con un listado de alimentos y bebidas más consumidos, reportados por un grupo representativo de mexicanos como resultado de la aplicación de la ENSANUT $2012^{19}$. El listado de los alimentos y bebidas más consumidos según la ENSANUT 2012 originalmente fue analizado por 100 gramos de porción de alimento, tomando como referencia la base de datos de información nutrimental del Departamento de Agricultura de los EE.UU. (USDA) ${ }^{20} y$ las Tablas de Valor Nutritivo de Alimentos Mexicanos $^{21}$. En caso de faltantes, también se utilizó el Sistema Mexicano de Alimentos Equivalentes $(S M A E)^{22}$ (Tabla 1). En el cuestionario de alimentación se busca incluir también una lista de alimentos industrializados, en los que, a diferencia del listado de alimentos y bebidas, la información se obtendrá de la etiqueta nutrimental incluida en su presentación comercial.

- Segunda etapa: fotografías. El acceso a fotografías para el cuestionario alimentario genera una aproximación única, ya que la descripción solo verbal o escrita está expuesta a interpretación y error. La estimación visual ofrece diferentes tamaños de porciones de alimentos frecuentemente consumidos, y está ligada al peso en gramos y a información nutrimental (Fig. 4). La selección de fotografías se realizó de acuerdo con trabajos previos en México, destacando el trabajo de la Universidad de Guadalajara, el Álbum fotográfico de alimentos mexicanos, que incluye fotografías

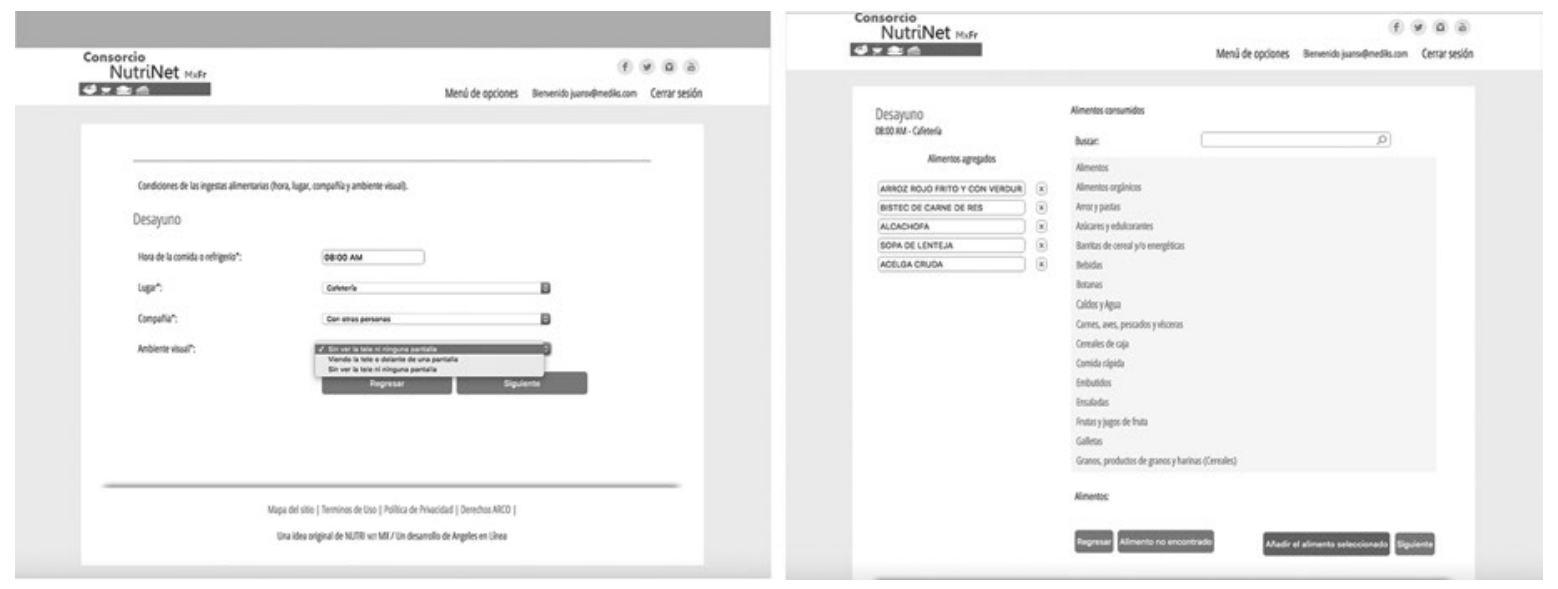

Figura 3. Imágenes que muestran la forma en que se irán detallando los alimentos consumidos en 24 horas. 
Cirugía y Cirujanos. 2018;86

Tabla 1. Integración de la base de datos de alimentos de Nutrinet-Salud México

\begin{tabular}{|c|c|c|c|}
\hline Base de datos & Aportación & Datos & Comentarios \\
\hline Alimentos y platillos mexicanos & INCMNSZ & $\begin{array}{l}\text { Datos de composición nutrimental medida en } \\
\text { alimentos y platillos mexicanos }\end{array}$ & $\begin{array}{l}\text { Datos por publicar del INCMNSZ } \\
\text { Es la única fuente mexicana de análisis } \\
\text { nutrimental directo de alimentos y platillos }\end{array}$ \\
\hline $\begin{array}{l}\text { Alimentos y platillos reportados } \\
\text { en ENSANUT }\end{array}$ & INSP & $\begin{array}{l}\text { Lista de } 1217 \text { alimentos y platillos } \\
\text { Análisis nutrimental (USDA o INCMNSZ 2007) }\end{array}$ & $\begin{array}{l}\text { Aumenta el error por cálculo de composición } \\
\text { nutrimental y uso de base de datos de los } \\
\text { EE.UU. }\end{array}$ \\
\hline $\begin{array}{l}\text { Recetas de platillos reportados } \\
\text { ENSANUT }\end{array}$ & INSP & $\begin{array}{l}\text { Lista de } 322 \text { platillos con receta seleccionada y } \\
\text { estandarizada, y su análisis nutrimental (USDA } \\
\text { o INCMNSZ 2007) }\end{array}$ & $\begin{array}{l}\text { Aumenta el error por selección de recetas y } \\
\text { cálculo de composición nutrimental y uso de } \\
\text { base de datos de los EE.UU. }\end{array}$ \\
\hline Alimentos del SMAE & SMAE & $\begin{array}{l}\text { Lista de alimentos del SMAE con análisis } \\
\text { nutrimental de diferentes fuentes }\end{array}$ & $\begin{array}{l}\text { Aumenta el error por cálculo } \\
\text { No todos los nutrimentos de todos los } \\
\text { alimentos están analizados } \\
\text { Bases de composición de alimentos variadas } \\
\text { para cálculo }\end{array}$ \\
\hline
\end{tabular}

ENSANUT: Encuesta Nacional de Salud y Nutrición; INCMNSZ: Instituto Nacional de Ciencias Médicas y Nutrición Salvador Zubirán; INSP: Instituto Nacional de Salud Pública; SMAE:

Sistema Mexicano de Alimentos y Equivalentes; USDA: Departamento de Agricultura de los EE.UU.

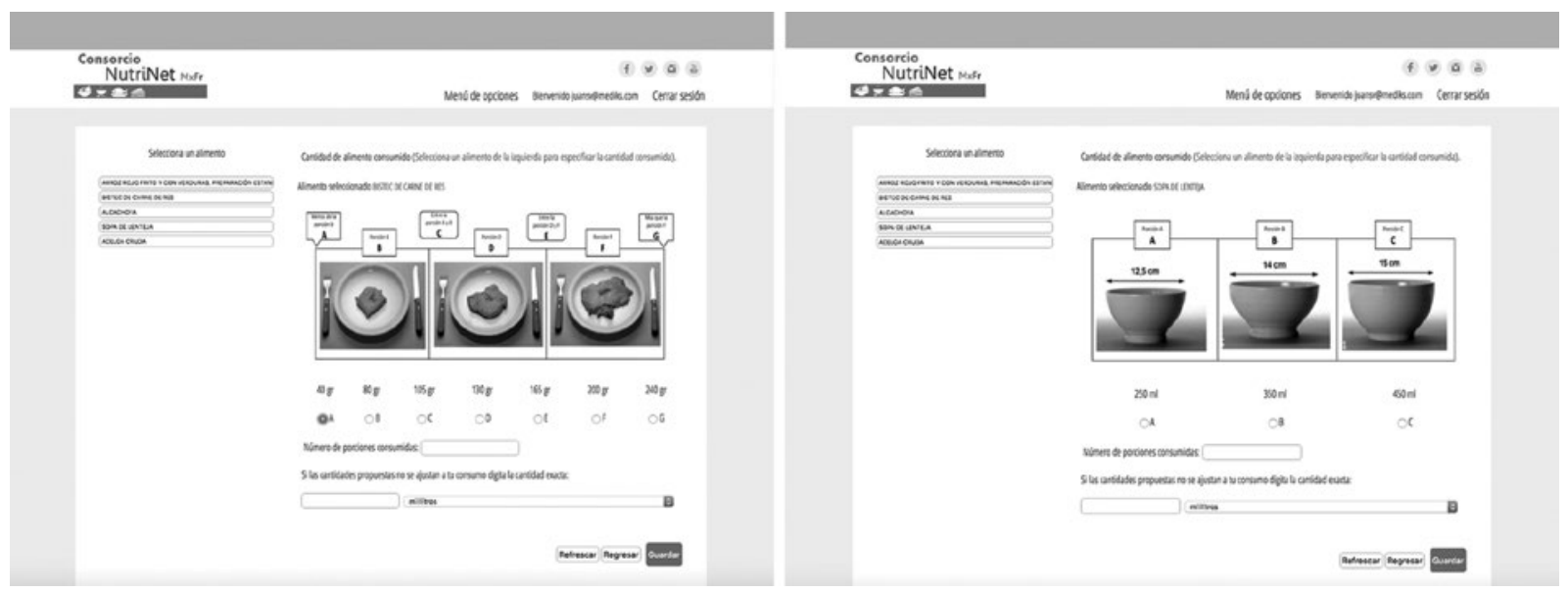

Figura 4. Imágenes que muestran la presentación visual de las porciones de alimentos consumidos.

de 359 alimentos ${ }^{23,24}$. La metodología utilizada para la elaboración de las fotografías fue valida$\mathrm{da}^{25}$ previamente y es similar a la de NutriNet-Santée ${ }^{6}$. Las fotografías incluidas en el cuestionario alimentario se complementaron con las de los alimentos del SMAE, nunca utilizadas ni publicadas, al igual que con algunas del sitio original de NutriNet-Santé que podían utilizarse en México (Tabla 2). Con la información disponible, se realizó una revisión detallada de la lista de alimentos y bebidas que cuentan con fotografía, y como resultado, NutriNet-Salud México incluye por primera vez en México y América Latina una base de datos de alimentos y bebidas con fotografía asociada a la información nutrimental resultado de la codificación en gramos de cada foto de porción o porción estándar reportada. Los alimentos o bebidas que se consideró que no requerían fotografía representativa se incluyeron en la plataforma asignando el gramaje de las porciones estándar que el usuario puede reportar (piezas, tazas, etc.). De la lista de alimentos y bebidas se determinó que algunos faltantes sí requerían fotografía $(n=34)$; siguiendo la misma metodología que en Francia (tres sesiones: un pilotaje y dos sesiones fotográficas), se realizó la toma de fotografías.

- Tercera etapa: flujo de la información y estructura del cuestionario y página de Internet. Se revisó el cuestionario de alimentación de NutriNet-Santé para respetar la misma estructura en la plataforma de México. Se revisaron las categorías de alimentos (p. ej., carnes, etc.) que se despliegan cuando el usuario registra una comida (p. ej., desayuno, comida, cena, colación), así como las subcategorías (p. ej., carne de res, de puerco, 
Tabla 2. Fuentes de fotografías de porciones de alimentos/platillos

\begin{tabular}{llll}
\hline Fotografías & Aportación & Detalles & Comentarios \\
\hline $\begin{array}{l}\text { Fotografías de alimentos } \\
\text { mexicanos }\end{array}$ & UDG & $\begin{array}{l}\text { Fotos de } 359 \text { alimentos y bebidas, } \\
\text { mostrando cuatro tamaños de porciones } \\
\text { diferentes }\end{array}$ & $\begin{array}{l}\text { Provienen de un manual para estimación } \\
\text { de porciones (aún por publicar) }\end{array}$ \\
$\begin{array}{l}\text { Fotografías de alimentos y } \\
\text { platillos mexicanos }\end{array}$ & SMAE & $\begin{array}{l}533 \text { fotografías mostrando diferentes } \\
\text { equivalentes de alimentos y platillos }\end{array}$ & $\begin{array}{l}\text { La mayoría de las fotos se tomaron como } 1 \\
\text { equivalente, 2 equivalentes o } 1 / 2 \text { equivalente }\end{array}$ \\
$\begin{array}{l}\text { Fotografías de alimentos y y } \\
\text { platillos de Francia }\end{array}$ & NutriNet-Santé Francia & $\begin{array}{l}\text { Se utilizaron } 89 \text { fotografías de las originarles } \\
\text { del cuestionario francés }\end{array}$ & $\begin{array}{l}\text { Solo se consideraron aquellos alimentos } \\
\text { que son similares o iguales en Francia }\end{array}$ \\
\hline
\end{tabular}

SMAE: Sistema Mexicano de Alimentos y Equivalentes; UDG: Universidad de Guadalajara.

ave, pescados y mariscos, etc.) dentro de cada categoría general. Aunque la plataforma debe ser casi idéntica a la plataforma de NutriNet-Santé, tomando en cuenta la cultura y los hábitos de alimentación en México se realizaron algunas adaptaciones. Se llevará a cabo un estudio piloto para el uso de este cuestionario dentro de la plataforma, que permitirá realizar ajustes y cambios conforme se requieran. Finalmente, se validará comparando su desempeño con la aplicación en persona del R24, en una muestra suficiente de individuos.

\section{Elaboración de los instrumentos éticos y jurídicos}

NutriNet-Salud México, para lograr su objetivo principal, requiere que el usuario, denominado «nutrinauta», ingrese en la plataforma una serie de información que, de acuerdo con nuestras leyes, en México se consideran datos personales y están clasificados como sensibles. Para ello, la información recabada a través de la plataforma NutriNet-Salud México da cumplimiento a las especificaciones definidas en las leyes en México, y previamente al llenado de la información y su envío el nutrinauta tendrá conocimiento y deberá aceptar el Consentimiento Informado y el Aviso de Privacidad, además de ser libre en todo momento de retirarse del estudio. Cabe señalar que la realización de este estudio fue aprobada por el Comité de Investigación y el Comité de Ética en Investigación del Instituto Nacional de Ciencias Médicas y Nutrición.

\section{Programación y construcción de las bases de datos}

La plataforma NutriNet-Salud México, para poder cumplir su función, requiere la captura y el almacenamiento de datos tomados de una serie de cuestionarios llenados por el sujeto en observación. El objetivo es recuperar esta información y poder realizar procesos con ella. Para lograr esta funcionalidad se diseñó un sistema de bases de datos relacionales basado en los siguientes conceptos: 1) estandarización de la información captada por los distintos cuestionarios; 2) captura de los cuestionarios de tal manera que se facilite la recuperación de su información, lo que llevó al grupo al diseño de un sistema de bases de datos relacional que permite la captura de los seis cuestionarios originales (el kit base más el cuestionario de inscripción), y tiempo después se anunció una tercera restricción que no estaba considerada; y 3) la adición permanente de nuevos cuestionarios.

La adición permanente de nuevos cuestionarios fue una restricción no considerada que llevó al grupo a cuestionar el diseño original, ya que la inclusión de cuestionarios nuevos significaba modificaciones no triviales en el diseño de la base de datos. Se propuso entonces generar un modelo de la base de datos partiendo de un nivel más abstracto, con el manejo del diccionario como si fuera otra estructura de datos que ingresa al sistema, de tal manera que permitiera la inclusión de cuestionarios nuevos sin modificaciones sustantivas en el sistema. Esto llevó a la generación de un modelo innovador, en el cual se modeló cada diccionario como una secuencia de tripletas: $<$ pregunta, tipo, respuestas_posibles >; esta abstracción, aunque agregó complejidad al diseño de la base de datos, permitió un manejo más adecuado de la estructura de cada diccionario.

En el sistema actual, los cuestionarios nuevos representan una entrada más para el sistema, que de manera automática administra su captura y recuperación de la información, y además brinda la posibilidad de analizar la información capturada con técnicas de big data, y enriquecida con herramientas de visualización que permitan observar la forma en que se agrupa y dispersa la información. 
Considerando el tipo de proyecto, uso, necesidades de transacción y almacenamiento, y posibilidades de integración con otras plataformas, NutriNet-Salud México utiliza MySQL ${ }^{25}$ (sistema de gestión de base de datos libre) y el paquete MySQL-admin (entorno gráfico de administración, auxiliar en la gestión de software, bases de datos, usuarios, recursos y seguridad).

\section{Creación de la página web y la interfaz}

En cuanto al desarrollo y la promoción del proyecto y el registro de los participantes (http://www.mexico-francia.mx/NutriNet/y http://www.NutrinetSaludMexico.mx), se propuso e implementó un esquema similar al de la versión pionera francesa, con el cual se permite realizar una mejor interacción usuario-servidor, considerando las tendencias de navegación que se utilizan de manera global actualmente.

Según los resultados de la encuesta sobre aprovechamiento de las tecnologías de la información y comunicaciones en los hogares de México, realizada por el Instituto Nacional de Estadística y Geografía en el año 2012, el $32.2 \%$ de los hogares del país contaba con computadora, porcentaje que se incrementó un $8.9 \%$ en un año. Asimismo, el $26 \%$ de los hogares contaba con una conexión a Internet, mostrando un crecimiento del $13.4 \%$ respecto a 2011. En 2014 se reporta un total de 53.9 millones de usuarios, que representan el $51 \%$ de la población, con un crecimiento anual del $5.3 \%$. Ciudad de México tiene el $60 \%$ del total de su población con acceso a Internet, y el $70 \%$ de los usuarios de Internet tienen 18 años de edad o más. Además, el total de las entidades gubernamentales y educativas de nivel superior tienen acceso a Internet ${ }^{26}$. Lo anterior, para un sistema de información en salud basado en el uso de Internet, como es NutriNet-Salud México, ofrece una gran oportunidad de acceder a una representativa muestra poblacional a un bajo costo y en tiempo real.

Una vez concluida la etapa de desarrollo, se iniciará la puesta en marcha de la plataforma:

- Etapa de ejecución institucional, durante 6 meses, en las instituciones y los dos sistemas de salud de los Estados que participan en NutriNet-Salud México, personal de salud y de educación, alumnos, pacientes y sus familias, con un corte para el análisis de datos, una publicación científica y recomendaciones en salud pública.

- Posterior a los 6 meses, lanzamiento de ámbito nacional de grandes campañas en diferentes medios de comunicación para el reclutamiento de voluntarios.

- Etapa de desarrollo de un supermercado virtual y realización de un ensayo aleatorizado del impacto de los sistemas de información nutricional.

\section{Resultados}

NutriNet-Salud México es un estudio de cohorte prospectivo, programado para un periodo de 10 años (2018-2028) mediante el uso de un sitio Web dedicado donde, una vez que el «nutrinauta» complete los cuestionarios, la información obtenida en la plataforma permitirá:

- Desarrollo de investigación aplicada en el campo: estudiar las relaciones entre los aportes en energía, nutrimentos, alimentos y conductas alimentarias, y la actividad física, con determinantes sociológicos, ecológicos, culturales, biológicos, etc., de las conductas alimentarias, del estado nutricio y del estado de salud; monitoreo de la evolución y tiempos, de consumo alimentario, actividad física y problemas nutricios y de salud de la población; calidad de vida; trastornos de la conducta alimentaria, depresión, deterioro cognitivo, etc.; incidencia de sobrepeso y obesidad, hipertensión, síndrome metabólico, cáncer, DM2 y ECV; mortalidad global y específica (para cáncer, DM2, ECV y relacionadas); y comprensión de los determinantes y comportamientos alimentarios y de la nutrición.

- El diagnóstico (nutrición y actividad física) como base del desarrollo de intervenciones y estrategias, para promover un estilo de vida saludable en la población.

- Evaluar el impacto de acciones de salud pública sobre el comportamiento alimentario y el estado nutricional, campañas, programas, etc. (en términos de conocimiento, percepción y eficacia).

- Comparación de la población de México, Institutos Nacionales, Universidades y Estados, con Francia, Bélgica y Suiza.

Es importante señalar que una de las grandes fortalezas de Nutrinet-Salud México es que ha permitido la integración de múltiples organismos e instituciones relacionadas con la salud en México, y que compartir información -como en este caso la relacionada con las bases de datos de alimentos, incluido su valor nutrimental, composición e imágenes, del cual no existía precedente en nuestro país-, ha sido un gran paso para este proyecto de investigación, respetando 
el modelo francés, que es la base para la creación del sistema, pero enfatizando su adaptación a lo que comemos los mexicanos.

Se unificaron bases de datos, en primer lugar la del INSP, que surgió de ENSANUT 2012 y que incluye los alimentos consumidos reportados por un grupo representativo de mexicanos durante esta Encuesta Nacional. Por otro lado, el Instituto Nacional de Ciencias Médicas y Nutrición Salvador Zubirán (INCMNSZ) terminó en 2016 la actualización de las Tablas de composición nutrimental de alimentos mexicanos, que han sido compartidas para poder utilizarse como parte del cuestionario de alimentación de Nutrinet-Salud México. De esta segunda base de datos, algunos nutrimentos fueron medidos directamente en los alimentos, y en aquellos faltantes se completó la información con la base de datos del USDA. En cuanto a los platillos referentes a la publicación ¿Qué y cómo comemos los mexicanos? Consumo de alimentos en la población urbana ${ }^{27}$, estudio gracias al apoyo del Fondo Nestlé, de FUNSALUD, el INSP realizó una base de datos de recetas para cada uno de ellos, para facilitar así su análisis nutrimental. Nutrinet-Salud México incluye algunas de estas recetas y complementa la lista de platillos. Se realiza un análisis de recetas comunes mexicanas con el programa de cómputo Food Processor SQL, que permite incluir ingredientes mexicanos o seleccionar de una amplia lista de alimentos y bebidas; para complementar la información se revisó el Sistema Mexicano de Alimentos. En cuanto a los alimentos industrializados, aunque las bases de datos citadas anteriormente incluyen varios, en el cuestionario de alimentación de Nutrinet-Salud México se completó la lista utilizando la información nutrimental de la etiqueta del producto, incluyendo diferentes marcas comerciales.

Así, el cuestionario de alimentación de Nutrinet-Salud México, como primer resultado de esta plataforma, incluirá la base de datos más completa de alimentos, platillos y bebidas, que podrá analizar el contenido nutrimental de acuerdo con las principales fuentes disponibles en la actualidad.

\section{Discusión}

El propósito de este proyecto es contar con información sobre la nutrición y los hábitos de salud de la población a través de un modelo de e-epidemiología, de un "sistema de información de salud", que aportará datos relevantes para el diseño de políticas de salud pública en México mediante: 1) proporcionar evidencia científica para definir las recomendaciones de salud pública nutricional adaptada a las diferentes poblaciones; 2) la identificación de las barreras para tomar medidas y guiar la definición y los términos de las acciones de salud pública; y 3) probar experimentalmente el impacto de las intervenciones de salud pública antes de su aplicación o como parte de las medidas introducidas en la población (impacto en términos de conocimiento, percepción, eficiencia, cambios de comportamiento y prácticas).

La implementación de NutriNet-Salud México permitirá en el corto plazo obtener información sobre los riesgos relacionados con la nutrición y los factores de protección, identificar los determinantes del estado nutricional y sus interacciones, y comparar los resultados con sistemas semejantes en Francia, Bélgica y Suiza. Además, a partir de la experiencia mexicana también se podrá compartir la plataforma con países de América Latina, siendo algunos de los interesados Colombia, El Salvador y Nicaragua, por mencionar algunos.

NutriNet-Salud México es el resultado de varios meses de trabajo en equipo de quienes conforman la RED de instituciones participantes, agregando valor a la plataforma a través de protocolos de investigación sobre desenlaces de la nutrición, sumando la experiencia de diferentes grupos de trabajo para conformar una base de datos completa y general de alimentos mexicanos con información nutrimental, complementada con fotografías de porciones.

\section{Agradecimientos}

A los co-autores e integrantes de RED NutriNet-Salud México: M. Abdo (AMC), S. Barquera (INSP, México), A. Basdevant (CHU Pitié Salpêtrière, París, Francia), T. Baubet (Université Sorbonne Paris 13 Cité, París, Francia), J.A. Barriguete Meléndez (INCMyN SZ, CEFM, AMC), J. Baulieux (Académie Nationale de Médecine, París, Francia), A.C. Benhammou (Ministère de la Recherche et de l'Enseignement Supérieur, París, Francia), M.F. Bernal-Orozco (Universidad de Guadalajara, México), R.H. Bourges (INCMNSZ, México), J. Dávila Torres (IMSS, México), C. Aranza (Secretaría de Salud, Estado de Michoacán de Ocampo, México), M.F. Cachera (Université Sorbonne Paris Cité, París, Francia), Z.I.L. Canela (Instituto Nacional de Cardiología, México), J.A. Cardona (Instituto Nacional de Perinatología, México), F. Cruz-Vega (IMSS; Academia Mexicana de Cirugía, México), F. Chico (Consejo Estratégico Franco-Mexicano, México), R.E. 
Colin (Instituto Nacional de Cardiología, México), T.J. Dávila (Academia Mexicana de Cirugía, México), S. Elizondo-Argueta (IMSS, México), P. Galán (Université Sorbonne Paris 13 Cité, París, Francia), M.C. Gómez (Secretaría de Salud del Estado de México, México), H.J.A. González (Instituto Nacional de Cardiología, México), L.M. Gutiérrez (Instituto Nacional de Geriatría, México), R.J.F. González (Secretaría de Salud Gobierno Federal, México), S Hercberg (Université Sorbonne Paris 13 Cité, París, Francia), M. Hernández-Ávila (INSP, México), L.G. Hernández (Fundación Franco-Mexicana para la Medicina IAP; Secretaría de Salud del Estado de Colima, México), B. Jácome (Universidad Autónoma de Guadalajara, México), N.E. Jaramillo (Secretaría de Salud Gobierno Federal, México), D. Kershenobich (INCMNSZ, México), P. Kuri-Morales (Secretaría de Salud Gobierno Federal, México), E.A. Lara (Secretaría de Salud del Estado de Colima, México), E.G. Leal (Instituto Nacional de Cardiología, México), T. Le Luong (Institut National de Prévention et d'Éducation pour la Santé, Francia), F.L. López (Instituto Nacional de Cardiología, México), K.M. López (Secretaría de Salud del Estado de México, México), M.A. Martínez Ríos (Instituto Nacional de Cardiología, México), R.M. Madero (Instituto Nacional de Cardiología, México), A. Meneses (Instituto Nacional de Cancerología, México), L. Magaña (INSP, México), M.A. Medina Mora (Instituto Nacional de Psiquiatría, México), M.A. Mejía (INSP, México), L.J. Morales (INCMNSZ, México), M.R. Moro (Maison de Solenn, Université de Paris Descartes, Sorbonne Paris Cité, Francia), J.M. Oppert (CHU Pitié Salpêtrière, París, Francia), B. Palacios-González (Universidad Iberoamericana, México), O. Perichart (Instituto Nacional de Perinatología, México), A.B. Pérez-Lizaur (Universidad Iberoamericana, México), S.R. Posadas (Instituto Nacional de Cardiología, México), R.C. Posadas (Instituto Nacional de Cardiología, México), R. Ridaura (INSP, México), G.F.J. Roldán (Instituto Nacional de Cardiología, México), J. Tapia (AMC), A.M. Vallejo (Instituto Nacional de Cardiología, México), B. Vizmanos (Universidad de Guadalajara, México), y C.A. Aguilar Salinas (INCMNSZ, México).

\section{Responsabilidades éticas}

El presente proyecto cuenta con la aprobación del comité científico y de ética del INCMyN Referiencia 1965 vigencia hasta el 25 de julio 2018.
Protección de personas y animales. Los autores declaran que para esta investigación no se han realizado experimentos en seres humanos ni en animales.

Confidencialidad de los datos. Los autores declaran que han seguido los protocolos de su centro de trabajo sobre la publicación de datos de pacientes.

Derecho a la privacidad y consentimiento informado. Los autores declaran que en este artículo no aparecen datos de pacientes.

\section{Financiamiento}

El presente estudio no recibió financiamiento alguno para su realización. Cada uno de los autores dedicó su trabajo sin recibir subsidio alguno.

\section{Conflicto de intereses}

No existen conflictos de intereses por parte de ninguno de los autores.

\section{Bibliografía}

1. World Health Organization. Noncommunicable diseases country profiles 2014. New York: WHO; 2014.

2. Olaíz G, Rivera Dommarco JA, Shamah T, Roja R, Villalpando S, Hernández Ávila M. Encuesta Nacional de Salud 2012. Cuernavaca, México: Instituto Nacional de Salud Pública; 2012.

3. García-García E, De la Llata Romero M, Kaufer-Horwitz M, Tusié-Luna MT, Calzada-León R, Vazquéz-Velázquez V, et al. La obesidad y el síndrome metabólico. Un reto para los Institutos Nacionales de Salud. Rev Invest Clin. 2009;61:333-46.

4. Álvarez del Río F, Gutiérrez-Delgado C, Guajardo-Barrón V. Costo de la obesidad: las fallas del mercado y las políticas públicas de prevención y control de la obesidad. En: Rivera Dommarco JA, Hernández Ávila M, Aguilar Salinas CA, Vadillo Ortega F, Murayama Rendón C, editores. Obesidad en México: recomendaciones para una política de Estado. México: UNAM; 2012. p. 279-88.

5. Ford ES, Bergmann MM, Kroger J, Schienkiewitz A, Weikert C, Boeing $\mathrm{H}$. Healthy living is the best revenge: findings from the European Prospective Investigation into Cancer and Nutrition-Potsdam Study. Arch Intern Med. 2009;169:1355-62.

6. Deglaire A, Méjean C, Castetbon K, Kesse-Guyot E, Hercberg S, Schlich $\mathrm{P}$. Associations between weight status and liking scores for sweet, salt and fat according to the gender in adults (The NutriNet-Santé study). Eur J Clin Nutr. 2015;69:40-6.

7. Camilleri GM, Méjean C, Bellisle F, Andreeva VA, Sautron V, Hercberg S, et al. Cross-cultural validity of the Intuitive Eating Scale-2. Psychometric evaluation in a sample of the general French population. Appetite. 2015;84:34-42.

8. Szabo de Edelenyi F, Julia C, Courtois F, Méjean C, Péneau S, Galan P, et al. Starchy food consumption in French adults: a cross-sectional analysis of the profile of consumers and contribution to nutritional intake in a web-based prospective cohort. Ann Nutr Metab. 2014;64:28-37.

9. Méjean C, Szabo de Edelenyi F, Touvier M, Kesse-Guyot E, Julia C, Andreeva VA, et al. Motives for participating in a web-based nutrition cohort according to sociodemographic, lifestyle, and health characteristics: the NutriNet-Santé cohort study. J Med Internet Res. 2014;16:e189.

10. Estrategia Nacional para la Prevención y el Control del Sobrepeso, la Obesidad y la Diabetes. México: Secretaría de Salud; 2013.

11. Hercberg S, Castetbon K, Czernichow S, Malon A, Mejean C, Kesse E, et al. The NutriNet-Santé Study: a web-based prospective study on the relationship between nutrition and health and determinants of dietary patterns and nutritional status. BMC Public Health. 2010;10:242.

12. Touvier M, Kesse-Guyot E, Méjean C, Pollet C, Malon A, Castetbon K, et al. Comparison between an interactive web-based self-administered $24 \mathrm{~h}$ dietary record and an interview by a dietitian for large-scale epidemiological studies. Br J Nutr. 2011;105:1055-64. 
13. Hercberg S, Castetbon K, Czernichow S, Malon A, Mejean C, Kesse E, et al. The NutriNet-Santé Study: a web-based prospective study on the relationship between nutrition and health and determinants of dietary patterns and nutritional status. BMC Public Health, 2010;10:242-6.

14. Deglaire A, Méjean C, Castetbon K, Kesse-Guyot E, Hercberg S, Schlich P. Associations between weight status and liking scores for sweet, salt and fat according to the gender in adults (The NutriNet-Santé study). Eur J Clin Nutr. 2015:69:40-6.

15. Lassale C, Péneau S, Touvier M, Julia C, Galan P, Hercberg S, et al. Validity of web-based self-reported weight and height: results of the Nutrinet-Santé study. J Med Internet Res. 2013;15:e152.

16. Medina C, Barquera, S, Janssen I. Validity and reliability of the International Physical Activity Questionnaire among adults in Mexico. Rev Panam Salud Publica. 2013;34:21-8.

17. Conway JM, Ingwersen LA, Moshfegh AJ. Accuracy of dietary recall using the USDA five-step multiple-pass method in men: an observational validation study. J Am Diet Assoc. 2004;104(4):595-603.

18. Gutiérrez JP, Rivera-Dommarco J, Shamah-Levy T, Villalpando-Hernández S, Franco A, Cuevas-Nasu L, et al. Encuesta Nacional de Salud y Nutrición 2012. Resultados Nacionales. Cuernavaca, México: Instituto Nacional de Salud Pública; 2012.

19. U.S. Department of Agriculture, Agricultural Research Service, USDA Nutrient Data Laboratory. USDA National Nutrient Database for Standard Reference, Release 24. USDA; 2011.
20. Ledesma JA, Chávez VA, Pérez-Gil RF, Mendoza ME, Calvo CC. Composición de alimentos. 2. ${ }^{\text {a }}$ ed. México: McGraw Hill; 2010.

21. Pérez-Lizaur AB, Palacios-González B, Castro AL, Flores Galicia I. Sistema mexicano de alimentos equivalentes. 4. ${ }^{\text {a }}$ ed. México: Fomento de Nutrición y Salud; 2014

22. Vizmanos-Lamotte B, López-Uriarte $P$, Hunot-Alexander $C$, Bernal-Orozco MF, Rodríguez-Rocha NP, Macedo-Ojeda G, et al. Álbum fotográfico de alimentos mexicanos. Guadalajara, Jal. Ediciones de la Noche; 2015.

23. Bernal-Orozco MF, Vizmanos-Lamotte B, Rodríguez-Rocha NP, Macedo-Ojeda G, Orozco-Valerio M, Rovillé-Sausse F, et al. Validation of a Mexican food photograph album as a tool to visually estimate food amounts in adolescents. Br J Nutr. 2013;109:944-52.

24. Hercbeg S, Deheeger M, Preziosi P. Portions alimentaires: manuel photos pour l'estimation des quantités. 3ème ed. Paris: SU.VI.MAZ-Candia/ Polytechnica; 2002.

25. MySQL Editions. Disponible en: https://www.mysql.fr/products

26. Instituto Nacional de Geografía y Estadística. México; 2012. Disponible en: http://www.inegi.gob.mx/est/contenidos/espanol/temas/Sociodem/ notatinf212.asp

27. Arvizu Martínez O, Polo Oteyza E, Shamah Levy T. Qué y cómo comemos los mexicanos. Consumo de alimentos en la población urbana. Instituto Nacional de Salud Pública. Fundación Mexicana para la Salud/ Fondo Nestlé para la Alimentación. México: Grafia Editores; 2015. 